\title{
Abstraction in Recovery Management
}

\author{
$J$ Elzot B Moss \\ Department of Computer and Information Science \\ University of Massachusetts \\ Amherst, Massachusetts 01003 \\ Nancy $D$ Griffeth \\ Marc $H$ Graham
}

School of Information and Computer Science

Georgia Institute of Technology

Atlanta, Georgia 30332

\begin{abstract}
There are many examples of actions on abstract data types which can be correctly implemented with nonserializable and nonrecoverable schedules of reads and writes We examine a model of multiple layers of abstraction that explains this phenomenon and suggests an approach to bulding layered systems with transaction oriented synchronization and roll back Our model may make it easier to provide the high data integrity of reliable database transaction processing in a broader class of information systems We concentrate on the recovery aspects here, a technical report [Moss et al 85] has a more complete discussion of concurrency control
\end{abstract}

\section{Introduction}

The database literature contains many examples of actions on abstract data types which can be correctly implemented with nonserializable schedules of reads and writes We mention one such example here

Example 1. Consider transactions $T_{1}$ and $T_{2}$, each of which adds a new tuple to a relation in a relational database Assume the tuples added have different keys A tuple add is processed by first allocating and filling in a slot in the relation's tuple file, and then adding the key and slot number to a separate index Assume that $T$,'s slot updating $\left(S_{j}\right)$ and index insertion $\left(I_{j}\right)$ steps can each be implemented by a single page read followed by a single page write (written $R T_{\jmath}, W T_{\jmath}$ for the tuple file, and $R I_{\jmath}, W I_{\jmath}$ for the index)

Here is an interleaved execution of $T_{1}$ and $T_{2}$

Permission to copy without fee all or part of this material is granted provided that the copies are not made or distributed for direct commercial advantage, the ACM copyright notice and the title of the publication and its date appear, and notice is given that copying is by permission of the Association for Computing Machinery To copy otherwise, or to republish, requires a fee and/or specific permission

(C) $1986 \mathrm{ACM} \quad 0-89791-191-1 / 86 / 0500 / 0072 \$ 0075$

$$
R T_{1}, W T_{1}, R T_{2}, W T_{2}, R I_{2}, W I_{2}, R I_{1}, W I_{1}
$$

This is a serial execution of $S_{1}, S_{2}, I_{2}, I_{1}$ Now $I_{1}$ and $I_{2}$ clearly commute, since they are insertions of different keys to the index Furthermore, $I_{1}$ cannot possibly conflict with $S_{2}$, sunce they deal with entirely different data structures So the intermediate level sequence of steps is equivalent to the sequence $S_{1}, I_{1}, S_{2}, I_{2}$, which is a serial execution of $T_{1}, T_{2}$ We have demonstrated serializability of the original execution in layers, appealing to the meaning (semantics) of the intermediate level steps $\left(S_{\text {, }}\right.$ and $I_{\jmath}$ ) But note that the sequence we gave may be a non-serializable execution of $T_{1}, T_{2}$ in terms of reads and writes, since the order of accesses to the tuple file and the index are opposite If the same pages are used by both transactions, it will be a non-serializable execution It is instructive also to observe that the sequence $R T_{1}, R T_{2}, W T_{1}, W T_{2}, \quad$ is not serializable even by layers It does not correctly implement the intermediate operations $S_{1}$ and $S_{2}$

A similar observation, which has received less attention, applies to recovery from action fallure The following example is an illustration of this interesting phenomenon

Example 2. Consider $T_{1}$ and $T_{2}$ as defined above, but suppose that the index insertion steps $I_{1}$ and $I_{2}$ each require reading and possibly writing several pages (as they might, for example, in a B-tree) We now write $R I_{,}(p), W I_{\jmath}(p)$ for reading and writing index page $p$ Consider the following interleaved execution of $T_{1}$ and $T_{2}$

$$
\begin{aligned}
R T_{1}, & W T_{1}, R T_{2}, W T_{2}, \\
& R I_{2}(p), R I_{2}(q), W I_{2}(q), W I_{2}(r), W I_{2}(p), \\
& R I_{1}(p), W I_{1}(p)
\end{aligned}
$$

The pair of index page writes $W I_{2}(q), W I_{2}(r)$ may be interpreted as a page split This is serializable by layers, since at the level of the slot and index operations we are executing the sequence $S_{1}, S_{2}, I_{2}, I_{1}$, as in Example 1 But we encounter the following difficulty if we subsequently decide to abort $T_{2}$ The index insertion $I_{1}$ 
has seen and used page $p$, which was written by $T_{2}$ in its index insertion step If we attempt to reproduce the page structure which preceded the page operations of $T_{2}$, we will lose the index insertion for $T_{1}$ Worse yet, if $T_{1}$ continues trying to operate on the index based on what it has seen of $p$, the structural integrity of the index could be violated Thus it appears that we cannot reverse the page operations of $T_{2}$ without first aborting $T_{1}$ But there is still a way to reverse the index insertion of $T_{2}$, just by deleting the key inserted by $T_{2}$ Consider the following sequence

$$
S_{1}, S_{2}, I_{2}, I_{1}, D_{2}
$$

The illustrated schedule is clearly correct, as long as the keys inserted by $T_{1}$ and $T_{2}$ are distinct, because we do not care whether the original page structure has been restored We only need to restore the absence of the key in the index

In this work, we present generalizations of serialızability and atomicity which account for many such examples The generalization arises from the observation that a transaction (or atomic action) is frequently a transformation on abstract states which 18 implemented by a sequence of actions on concrete states The usual definttion of serializability requires equality of concrete states We call this concrete serzalzzability, to distinguish it from equality of abstract states, which we call abstract serialızabılıty Since many different concrete states in an implementation may represent the same abstract state, abstract serializability is a less restrictive correctness condition than concrete serializability An immediate apphcation of abstract serializability is to explain the correctness of apparently nonserializable schedules such as those described in [Schwarz and Spector 84] and [Weihl 84] If results returned by actions are considered part of the state, correctness conditions for read only transactions, such as those described in [Garcia and Wrederhold 82], can also be expressed Abstract serializability also explains the phantom record problem and generalizes the Idea of predicate locks as presented in [Eswaren et al 76]

It seems worthwhile to note here there abstract serializability, when applied to concurrency control via locking, deals only with the level of abstraction of locks, not with lock granularity Lockıng pages or tuples is physical locking and occurs at a lower level of abstraction than predıcate lockıng on relations Lockıng tuples, slots, byte ranges, pages, or files 18 all physical locking, but at different levels of granularity Similarly, locking individual keys, ranges of keys, columns, groups of columns, or predicates (suitably restricted to avold NP-complete computations) is all abstract locking, but at different granularities In short, granularity and level of abstraction are orthogonal concepts It may still be useful and desirable to offer several degrees of granularity of locking at any given level of abstraction

Level of abstraction has perhaps more to do with duration of locking than granularity Our theory unfies "short" locks, acquired to protect a data structure's integrity for a single manipulation and then released, with transaction locks, held until transaction completion, and in addition shows how intermediate duration locks can be used correctly

The generalization of atomicity is analogous to that of serializability The usual definition of an atomic action requires that it execute to completion or appear not to have happened at all We introduce the idea of abstract atomicity, which is analogous to abstract serializability A schedule of actions is abstractly atomic if it results in the same abstract state as some schedule in which only the non-aborted actions have run Concrete atomicrty corresponds to the more usual definition The final state is the same (concretely) as one that would have resulted from running only the concrete actions which were called by non-aborted abstract actions

A widely accepted folk theorem states that it is necessary to use knowledge of the semantics of actions to achieve more concurrency than serialization allows While we could address the semantics of specific atomic actions case by case, this is a tedious process Instead, we describe a systematic method of using easily obtained knowledge about their semantics A basic theorem of this paper, in a result related to the results of [Beer1 et al 83], says that we can serialize at the individual levels of abstraction Between levels, we need only to insure that the serialization order is preserved Thus, in the above example, once the slot manipulation has been completed, locks on the page and its internal allocation structure may be released It is not necessary to wait until $T_{1}$ is complete (We do need to retain a (more abstract) lock on the slot and opposed to the page) This has the effect of shortening transactions and thereby increasing concurrency and throughput The analogous result holds for atomicity we show that, for schedules which are serialızable by layers, atomicity need only be enforced within each level of abstraction

Another contribution is a much more realistic (but slightly more complicated) model than the usual straight line model of transactions (as presented, for example, in [Papadimitriou 79]) The model presented here accounts for the flow of control in programs, such as if-thenelse and while statements, without introducing nearly as much complexity as is present in [Beeri et al 83] The most interesting result involving the model is that, while it affects the classes of abstractly serializable and concretely serializable schedules in potentially profound ways, the class of conflict preserving serializable (CPSR) schedules (those that can be serialized by interchanging adjacent non-conflicting actions) is essentially the same 
This is because interchanges of non-conflicting actions preserves the flow of control withm an action as well as the resulting state It does not appear that any authors have previously addressed this issue

The definitions of abstract and concrete serialıability and atomicity do not suggest practical implementations. It is widely accepted, however, that the largest class of serializable schedules which is recognizable in any practical sense is the class of CPSR schedules A similar situation may hold for atomicity We define here a class of conflict-based atomic schedules which can be executed efficiently This is the class of restorable schedules, in which no action is aborted before any action which depends on it This class may be vewed as dual to the class of recoverable schedules defined in [Hadzila$\cos 83$ ] A schedule is recoverable if no action commits before any action which it depends on In a restorable schedule, aborts can be efficiently implemented by executing state-based undo actions for each child action of an aborted action

Finally, this work addresses a problem mentioned but not specifically addressed in (Beeri et al 83), which is the use of knowledge about abstract data types and state equivalence in serialization The "fronts" of [Beeri et al 83], which must be computed from an actual history of the system, can be determined in this context from information easily provided by a programmer namely, from the call structure of the system and a "may conflict predscate" which describes which actions may conflict (1 e, not commute) with each other The use of knowledge about abstractions and state equivalence permit descripton of legal interleavings in a simpler and more direct manner than in [Beeri et al. 83] or in the multilevel model of [Lynch 83], where the set of legal interleavings must be given directly

Similarly, the semantic information used for recovery can be provided easily by the programmer The undos must themselves be actions (which will have to be coded if they are not "natural" actions for the abstraction) In each action, there must be a case statement which specifies the undo action for each set of states For example, if the forward action is "Add key $x$ to index $I^{\text {" }}$ then for the set of index states in which the index does not already contain $x$, the undo is "Delete key $x$ from index In For the set of index states in which the index already contains $x$, the undo action is the identity action

In the presentation below, we have omitted proofs and some inessential detals Full proofs and discussion are provided for the recovery results See [Moss et al 85] for complete coverage of both concurrency and recovery

\section{The Model}

We first describe the model for a single level of abstraction The essential difference between this model and the straight line model used in [Papadımitriou 79] is that the flow of control 18 reflected in the model The essential difference between this model and those in [Beeri et al 83] and [Lynch 83] 1s that the construction of the set of legal interleavings is simple and visible in the model Some notation will be needed to describe the levels of abstraction

Notation: Let $S_{1}$ be an abstract state space and let $S_{0}$ be a concrete state space Let $A_{1}$ be a set of abstract actions and $A_{0}$ be a set of concrete actions Let $\rho \quad S_{0} \rightarrow S_{1}$ be a partial function from concrete to abstract states If $\rho(t)=s$ for concrete state $t$ and abstract state $s$, then $t$ represents $s$

The intuition is that concrete states are used to represent abstract states and concrete actions are used to implement abstract actions Not every concrete state represents a valid abstract state Furthermore, the same abstract state may be represented by several different concrete states However, we do expect that every abstract state is represented by some concrete state, that 1s, $\rho\left(S_{0}\right)=S_{1}$

Actions map states to states according to a meaning function The meaning function for a concrete (abstract) action is a function $m \cdot A_{0} \rightarrow 2^{S_{0} \times S_{0}}$ $\left(m A_{1} \rightarrow 2^{S_{1} \times S_{1}}\right)$ It is interpreted as follows. If $\langle s, t\rangle \in m(a)$ for an action $a$ then when executed on state $s$, the action $a$ can terminate in state $t$ Actions are nondeterministic, that 18 , there may be more than one terminal state $t$ for a given initial state $s$.

Abstract actions are implemented by programs over concrete actions These programs generate sequences of concrete actions We do not assume that any particular method of generating the sequences is used. In proofs, we assume only that each program is associated with a set of sequences of concrete actions, which is the set of sequences the program would generate when run alone, and that new programs can be constructed from existing programs by concatenation. This operation amounts to running the first program to completion and then initiating the second program Note that when two programs run concurrently, one or both of them may generate a sequence of actions that would not be generated if they ran alone Such sequences may be unacceptable

A single concrete action is a program, as is the concatenation of two programs If $\alpha$ and $\beta$ are programs, then the meaning of their concatenation $\alpha, \beta$ is to execute first $\alpha$ and then $\beta$ 


$$
\begin{aligned}
& m(\alpha ; \beta)=\{(s, t\rangle \mid \exists u .\langle s, u\rangle \in m(\alpha) \wedge \\
& \langle u, t\rangle \in m(\beta)\} \text {. }
\end{aligned}
$$

Since concatenation of actions is clearly associative, we write $\alpha_{1} ; \ldots ; \alpha_{n}$ for concatenation of $n$ programs, ignoring the order of concatenation

Notation: For any subset $C$ of $S_{0} \times S_{0}$ let

$$
\begin{aligned}
\rho(C)=\{\langle s, t\rangle \mid \exists\langle x, y\rangle \in C \cdot \rho(x) & =s \wedge \\
\rho(y) & =t\}
\end{aligned}
$$

We say that an abstract action 18 implemented by a program of concrete actions if $\rho$ maps the meaning of the concrete program to the meaning of the abstract action. We will also require that if the program is initiated in a valid state then it must terminate in a valid state.

Definition: $A$ concrete program $\alpha$ implements an abstract action $a$ if and only if

1. $m(a)=\rho(m(\alpha))$ and

2 for every pair $\langle a, b\rangle \in m(\alpha)$, if $\rho(a)$ is defined then $\rho(b)$ is also defined.

We now state a technical lemma about umplementations which will be useful in a subsequent section

Lemma 1: Let $a$ and $b$ be abstract actions implemented by concrete programs $\alpha$ and $\beta$, respectively Then $m(a, b)=\rho(m(\alpha, \beta))$

Corollary 1 to Lemma 1: Let $a$ and $b$ be abstract actions implemented by concrete programs $\alpha$ and $\beta$ Then the abstract action $c$ having $m(c)=m(a, b)$ can be implemented by the concrete program $\gamma=$ $\alpha ; \beta$

Corollary 2 to Lemma 1: Let $a_{1},, a_{n}$ be abstract actions implemented by concrete actions $\alpha_{1}, \ldots, \alpha_{n}$ Then the abstract action $c$ defined by $a_{1} ; . ; a_{n}$ can be implemented by the program $\alpha_{1} ; ; \alpha_{n}$

In keeping with the use of an intializing action in [Papadimitriou 79], we assume that the database has been initialized to concrete state $I$ in the domain of $\rho$ (i e., $\rho(I)$ is the initial abstract state). It will often be useful to restrict the meaning function to those paurs whose initial state is $I$.

Notation: The restricted meaning function for program $\alpha$ is defined

$$
m_{I}(\alpha)=\{\langle I, \jmath\rangle \mid\langle I, \jmath\rangle \in m(\alpha)\}
$$

The restricted meaning function for abstract action $a$ is defined

$$
m_{\rho(I)}(a)=\{\langle\rho(I), \rho(\jmath)\rangle \mid\langle\rho(I), \rho(\jmath)\rangle \in m(a)\}
$$

If $\alpha$ implements $a$ then $m_{\rho(I)}(a)=\rho\left(m_{I}(\alpha)\right)$ Associated with each program 18 a set of possible computations of the program, one for each sequence of concrete actions which can be executed to completion.

Definition: A computation of an abstract action a having program $\alpha$ is a sequence $C=c_{1}, \quad, c_{n}$ of concrete actions, in the set of such sequences defined by the program, such that $m_{I}(C)$ is nonempty

A computation of a set $a_{1}, ., a_{n}$ of concurrent abstract actions is an interleaving of the concrete actions in computations for $\alpha_{1}, \quad, \alpha_{n}$ which can be run to completion

Definition: A concurrent computation of the set $a_{1}, \quad, a_{n}$ of abstract actions is an interleaving $C$ of computations of the individual actions such that $m_{I}(C)$ is nonempty

\section{Serializable Computations}

\subsection{Serializability of Abatract Actions}

The set of concurrent computations for a collection of actions will in general be hard to characterize. It may be even harder to characterize the ones which are correct We discuss a relatively simple subset of these computations, those that behave, in some sense, like serial (noninterleaved) computations To completely describe an interleaved computation of some abstract actions, we introduce a structure called a log It includes the abstract actions whose execution is interleaved, the actual interleaved sequence of concrete actions, and an indication of which concrete actions were generated by (programs of) which abstract actions

Definition: $A \log L$ is a set $A_{L}$ of abstract actions, a sequence $C_{L}$ of concrete actions, and a mapping $\lambda_{L} \quad C \rightarrow A$ such that $\lambda_{L}(c)$ is the abstract action $a \in A_{L}$ on whose behalf $c$ is run $L$ is complete if $C_{L}$ is a concurrent computation of $A_{L}$, and partial if $C_{L}$ is a prefix of a concurrent computation of $A_{L}$.

Definitions are stated and results proved for complete logs unless otherwise indicated Usually, the extension to partial logs is trivial

Notation: $m\left(c_{1} ; ; ; c_{n}\right)$ may be written as $m\left(C_{L}\right)$ when $C_{L}=\left\langle c_{1}, \quad, c_{n}\right\rangle$ (a sequence where $c_{2}$ precedes $c_{\text {, }}$ for $\left.z<\jmath\right)$

Notation: We will write $c<_{L} d$ when $c$ precedes $d$ in the sequence $C_{L}$

We consider serial computations to be correct

Definition: Consider a $\log L$ containing abstract actions $A_{L}=\left\{a_{1}, \quad, a_{n}\right\}$ implemented by programs $\left\{\alpha_{1}, \quad, \alpha_{n}\right\}$ The $\log L$ is serral if $C_{L}$ is a computation of the program $\alpha_{\pi(1)}, \quad, \alpha_{\pi(n)}$ for some permutation $\pi$ of $\{1,, n\}$ 
We also consider a computation to be correct if it results in an abstract state that would result from some serial log The following defintion allows the use of knowledge about abstractions in determining the correctness of an interleaving Depending on the abstraction, this can be a very different class of interleavings from those that would ordinarily be viewed as serıalızable

Definition: A $\log L$ is abstractly serralizable if and only if there is a permutation $\pi$ of $\{1, n\}$ such that $\rho\left(m_{I}\left(C_{L}\right)\right) \subset m_{\rho(I)}\left(a_{\pi(1) ;}, a_{\pi(n)}\right)$

This says that the abstract effect of running the concrete actions in $C_{L}$ must be consistent with (though perhaps not include all possibilities of) executing the abstract actions in some order

The next definition defines a class of serializable logs more closely related to the usual class of serializable schedules

Definition: $A \log L$ is concretely serzalizable if and only if there is a permutation $\pi$ of $\{1, \quad, n\}$ such that $m_{I}\left(C_{L}\right) \subset m_{I}\left(\alpha_{\pi(1)}, \quad, \alpha_{\pi(n)}\right)$

Definition: For both abstract and concrete serializability, the sequence $\pi(1), \quad, \pi(n)$ is called the serzalization order of $L$

A partial $\log L$ is serial (concretely serializable, abstractly serializable) if there is a complete serial (concretely serializable, abstractly serializable) $\log M$ such that $C_{L}$ is a prefix of $C_{M}$, that 18, if $L$ can be extended (completed) to have the property in question

Concrete serializabllty, which requures that concrete states be the same, 18 more restrictive than abstract serualizability, which requires only that abstract states be the same

Theorem 1: If the $\log L$ is concretely serializable then it is abstractly serializable

This theorem can easily be extended to partial logs For a partial $\log L$ which is concretely serializable, there is a concretely serializable complete $\log M$ such that $C_{L}$ is a prefix of $C_{M}$ By the above theorem, $M$ is also abstractly serializable, hence $L$ is abstractly serializable

Concrete serializability is not identical to the class SR of serializable executıons as defined in [Papadımıtrıou 79] because of the nondetermmism and because it 18 necessary to check that the reordered collection of actions is a computation. If abstract actions are implemented only by straight line programs, as in [Papadimitriou 79], then any serial schedule of the concrete actions in a concurrent computation is still a computation But this is not the case in our model, because we allow transactions to make decisions as they run (represented by nondeterministic choice of concrete program for abstract action), and interleaving can affect decision making We cannot interchange actions of a computation arbitrarly and expect the result to remain a computation $A$ subsequent lemma gives one mechanism by which we can verify that a transformation of a computation is still a computation The key is to insure that a transaction's decisions are not affected by the concurrent execution of steps from other transactions

It should be noted that this model reduces to the model in [Papadimitrou 79] if the concrete actions are deterministic reads and writes with the obvious meanings assigned to them and if all programs are constructed by concatenation only It was shown in [Papadimitriou 79] for these concrete actions that concrete serializability is NP-complete Without more information about the semantics of the actions, however, and about the abstraction function, we cannot say anything about the complexity class of either concrete or abstract serializability

For this reason, neither abstract nor concrete serializablity has significance as a definition of a class of schedules which we can recognize However, abstract serializablity is a valuable correctness condition for explaning the correctness of schedules such as the one in the opening example In a subsequent section, we generalize this use of abstract seriahzability to explain the correctness of a large class of schedules, many of which are not concretely serializable But first, we translate another standard serializability result to the new model of program execution

Definition: Actions $a$ and $b$ commute of $m(a, b)=$ $m(b, a)$ Otherwise, $a$ and $b$ confluct

Definition: Let $C$ and $D$ be sequences of concrete actions We say that $C \approx D$ if they are identical except for interchanging the order of two nonconflicting concrete actions, that 18, actions $c$ and $d$ such that $m(c ; d)=m(d, c)$ The transitive, reflexive closure of $\approx 18$ denoted by $\approx *$

The following lemma provides the basic mechanism for establishing that a permuted computation is still a computation We use it to verify that a serial (noninterleaved) sequence of concrete actions could actually have been requested by the given atomic actions, that is, it is a semantically as well as syntactıcally valid sequence of actıons

Lemma 2: If $L$ is a $\log$ and if $D \approx{ }^{*} C_{L}$ and $D$ is constructed from $C_{L}$ by interchangung nonconflicting operations $c$ and $d$ such that $\lambda(c) \neq \lambda(d)$, then there is a $\log M$ with $A_{M}=A_{L}, C_{M}=D$ and $\lambda_{M}=\lambda_{L}$ Furthermore, $m\left(C_{L}\right)=m\left(C_{M}\right)$

In this lemma, $D \approx{ }^{*} C_{L}$ insures that the (concrete) meanings of $D$ and $C_{L}$ are the same, and the condition on $\lambda$ insures that $D$ is a concurrent computation of the 
abstract actions $A_{L}$, and is different from $C_{L}$ only in how the concrete actions are interleaved

Definition: Logs $L$ and $M$ are equivalent if $A_{L}=$ $A_{M}, \lambda_{L}=\lambda_{M}$, and $C_{L} \approx{ }^{*} C_{M}$ If $L$ is equivalent to $M$ for a serial $\log M$, then $L$ is conflict preserving serialzable (CPSR)

Theorem 2: If a $\log L$ is conflict preserving ser1alizable, then it is concretely serializable

Applying 1, we further conclude that a CPSR log is abstractly serializable There is nothing particularly surprising or new about 2 It is a restatement of famliar serializability results in our formalism

\subsection{Layered Serializability}

In this section, the definitions of serializability are extended to multiple levels of abstraction and the basic result on serializability is stated We make two sumplfying assumptions the levels of abstraction are totally ordered, and each action calls subactions belonging to the next lower level of abstraction only How to weaken these assumptions is discussed in [Moss et al 85] We assume a system with $n$ levels of abstraction First, let us introduce notation for the states and actions of an $n$ level system

Notation: The concrete state at level 2 is $S_{\imath-1}$ The abstract state is $S_{q}$ The abstraction mapping at level $\imath$ is $\rho_{2} \cdot S_{t-1} \rightarrow S_{i}$ The set of concrete actions is $C_{2}$ The set of abstract actions is $A_{8}=$ $\left\{a_{1}, \quad, a_{3, k_{2}}\right\}$ The number of abstract actions at level $\imath$ is $k_{t}$ Concrete actions at level $\imath$ are abstract actions at level $z-1$ Thus $C_{\imath}=A_{t-1}$

We need also to extend the notion of a log, which represents a particular concurrent execution of some abstract actions in the system, to $n$ level systems Given a collection $A_{n}$ of top level actions, concurrent execution of the actions is described as follows.

Definition: A complete system $\log \mathrm{L}$ is a collection of complete logs $\left(L_{1}, \quad, L_{n}\right\rangle$ such that $L_{4}$ is a complete log for level $\imath$ and the concrete actions in the $\log L_{1}$ are the same as the abstract actions in the $\log L_{s-1}$

In a complete system log we have in essence a forest of actions, with one tree rooted in each top level action However, the set of actions at each level (except the top) $1 s$ ordered (by the log at that level) Complete system logs have related partial logs

Definition: A partial system $\log \mathbf{L}$ is a collection of partsal logs $\left\langle L_{1}, \quad, L_{n}\right\rangle$ such that $L_{i}$ is a partial $\log$ for level 2 , and the concrete actions in the $\log L_{8}$ are a subset of the abstract actions in the $\log L_{z-1}$
Those concrete actions of $L_{2}$ that are not abstract actions in $L_{1-1}$ can be viewed as simply never (or not yet) undertaken at the next lower level

Definition: The top level $\log$ for a system $\log \mathrm{L}$ consists of the top level abstract actions $\left(A_{n}\right)$, the bottom level concrete actions $\left(C_{1}\right)$, and the mapping from concrete to abstract actions constructed by composing the $\lambda_{2}$, namely $\lambda_{1} \circ \circ \lambda_{n}$

A top level log gives a characterization of a system in terms of the overall effect of "user oriented" (that 1s, top level) actions on the "real state" of the system (contained in $S_{0}$ ), ignorng internal structure Our original examples showed that we have reason to believe that a large class of top level logs are correct even though they are not concretely serralizable In fact, our approach gives a characterization of an interesting, realizable subclass of executions whose top level logs are readily demonstrated to be abstractly serializable, though perhaps not concretely serializable

Definition: The system $\log \mathbf{L}$ is abstractly (concretely) serzalizable by layers if each $L_{t}$ is abstractly (concretely) serializable and there is a serialization order on $A_{t-1}$ which 18 the same as the total order on $C_{z}$ We will denote this serialization order $\pi_{z}$

The following theorem justifies the practice of "serialszing by layers", that 1s, providing serialization for the individual levels of abstraction and forgetting subaction conflicts (e $g$, releasing locks) as soon as the action at the next higher level is complete

Theorem 3: If a system $\log L$ is abstractly serializable by layers then its top level log is abstractly serializable

If $L$ is partial, then we can extend the sequence of concrete actions to a computation having the above properties Thus the result also holds for partial logs

Since concrete sernalizabılity of a layer implies abstract serializability of that layers, we readily derive a very useful result.

Corollary 1 to Theorem 3: If a system $\log \mathrm{L}$ is concretely serializable by layers, then its top level $\log$ is abstractly serializable

In practice, transaction concurrency control mechanisms enforce concrete serializability of a layer (the abstract actions are called transactions and the concrete actions are called sumply actzons or transaction steps) Hence, the traditional methods can be applied layer by layer to guarantee abstractly serialıable top level logs

Definition: If a system log is serializable by layers and if each $\log L_{t}$ is conflict preserving serializable, then the set of logs is called conflict preserving serzalzzable by layers (LCPSR) 
Since all practical serialization methods recognize only subsets of the set of CPSR logs, the following result is the interesting one, from the practical point of view

Corollary 2 to Theorem 3: If a system $\log L$ is conflict preserving serializable by layers then its top level log is abstractly serializable

Two phase locking is one way of achieving CPSR schedules, which is one of the things shown in [Eswaren et al 76] The corollary just stated demonstrates the correctness of performing two phase locking at each level of abstraction Ignoring the possibility of deadlock, the locking protocol that results is as follows

1 Prior to performing a (non-top level) action $a_{4}$, , acquire a lock appropriate to the operation and its arguments, which will prevent conflicting level $\imath$ operations from being initiated until the lock is released

2 As a level $\imath$ operation's program is executed, a number of level $\imath-1$ locks will be acquired, as a result of the preceding rule

3 When a level 2 operation completes ("commits"), release all level $\imath-1$ locks associated with its execution, but keep the level $\bullet$ lock to protect level $\imath+1$

In this protocol the duration of a level $\imath$ lock is from the time it is acquired until the completion of the level $\imath+1$ operation that caused it to be acquired If we have only two levels (transaction and action), this reduces to the usual locking for transactions, with appropriately abstract locks (e g, as in [Schwarz and Spector 84] or [Weihl 84])

\section{Recovery from Action Failure}

One method of enforcing serializability is to abort actions which violate serializability constraints, and every practical serialization technique sometimes uses aborts for this purpose Thus serialization implies the posstblity of action failure and it is necessary to guarantee correct recovery from failure to guarantee serialıability The converse is not true, and so we initially consider fallure atomicity without assuming serializablity

The rest of this paper discusses recovery from the fallure of a sungle action by eliminating its partial effects Two methods of elimmating partial effects are $\mathrm{m}$ common use One is to roll the action back by undorng each change it has made The other is to restore the system from a checkpoint taken prior to initialızation of the action, redoing each subsequent concrete action other than those called by the aborted action We develop the conditions which permit use of redos in section 41 This more general, though probably not practically appealing approach, is specialized in section 42 , where we examine the use of undos as used for transaction rollback in database systems In both sections, we assume a single level of abstraction Note that we are not addressing crash recovery, only transaction abort

In section 43 , the results are extended to a multilevel system and a result analogous to the result for layered serializability is stated Unlike a smgle layer system, with multiple layers serializability is required to establish that the required sequence of concrete actions in a level of abstraction was implemented by the next lower level

\subsection{Aborting Actions}

An abstract action is not inherently atomic, since it is implemented by a sequence of concrete actions If it fails after execution of some of the concrete actions, then the effects of those actions which have been completed must be elımmated The process of elımunating any partial effects of a falled abstract action will be referred to below as an abort of the action

We first formalize the meaning of aborting an action, without bias towards any particular notion of how to implement aborts We then introduce the notion of simple aborts those aborts that are equivalent to omitting the concrete effects of the aborted action Next we develop some termunology and notation regarding transaction dependencies, especially as they relate to aborts An important product of that discussion is a property called restorability, which is related to recoverability as discussed in [Hadzilacos 83] Finally, we prove a result relating restorability, simple aborts, and correctness

To abort an action properly, it is necessary to change the current state to a state equivalent to one in which the action was not executed at all Let LOGS be the set of all logs (Remember that a $\log L$ consists of a set $A_{L}$ of abstract actions, a sequence $C_{L}$ of concrete actions, and a mapping $\left.\lambda_{L} \quad C \rightarrow A\right)$ We define an operator which chooses a concrete abort action when it 18 given a log and abstract action to be aborted

$$
\text { ABORT LOGS } \times A \rightarrow\left(S_{0} \rightarrow S_{0}\right)
$$

The abort must restore some state consistent with executing the abstract actions in $A_{L}-\{a\}$

Definition: An action generated by the $A B O R T$ operator is called an abort An action is sald to be aborted if its last action 18 an abort (of itselt)

A log which contains aborts should appear to be a $\log$ which contains all of the non-aborted actions and none of the aborted actions We call such a log abstractly atomic Abstract atomicity is the fundamental correctness condition for aborts

Definition: A complete $\log L$ is abstractly atomic 
if there is a complete $\log M$ having the following properties

$$
\begin{array}{ll}
1 & A_{M}=A_{L}-\{a \mid a \text { is aborted in } L\} \text { and } \\
2 & \rho\left(m_{I}\left(C_{L}\right)\right) \subset \rho\left(m_{I}\left(C_{M}\right)\right)
\end{array}
$$

Note that we have not required that the $\operatorname{logs} L$ and $M$ be sernalizable Any computation is all right according to the above definition Later, to achieve "layered atomicity", we will assume serializability

Note also that abstract atomicity requires only that the resulting concrete stated be equivalent (under $\rho$ ) to one in which the aborted actions were not run That is, the second part of the definition does not imply anything about the relationship of $m_{I}\left(C_{L}\right)$ to $m_{I}\left(C_{M}\right)$ In some cases it may be useful to impose the stronger condition of concrete atomicity

Definition: A complete $\log L$ containing aborted actions is concretely atomic if it there is a complete $\log M$ having the following properties

$$
\begin{array}{ll}
1 & A_{M}=A_{L}-\{a \mid a \text { is aborted in } L\}, \\
2 & m_{I}\left(C_{L}\right) \subset m_{I}\left(C_{M}\right) .
\end{array}
$$

We extend the definition of atomicity to partial logs in the obvious way

Definition: A partial $\log L$ is abstractly (concretely) atomic if there is a complete abstractly (concretely) atomic $\log M$ such that $A_{M}=A_{L}, C_{L}$ is a prefix of $C_{M}$, and $\lambda_{L}$ is $\lambda_{M}$ restricted to $C_{L}$

It follows immediately from the definitions that concrete atomicity implies abstract atomicity

With definitions of atomicity accomplished, let us now consider how to achieve it One way to implement abstract atomicity is to restore state $I$ and rerun the actions in $A_{M}$ The state $I$ then serves as a checkpoint This redo approach is what we assume for now, rollback 1s discussed later. Note that an arbitrary choice of $M$ in the above defintion may require re-running the abstract actions, not just the concrete actions In an online, high volume transaction system, this is not a practical method The programs for the abstract actions may not even be available after they terminate In such a system, we want aborts to be simpler For this reason we wall require that the $\log M$ have a very simple relationship to the $\log L$ that $C_{M}$ be $C_{L}$ minus the children of aborted actions In this case, to abort $a$, we remove the effects of ats concrete steps $\lambda_{L}^{-1}(a)$ by restoring a final state for $m_{I}\left(C_{L}-\lambda_{L}^{-1}(a)\right)$

Notation: As long as it is clear what $\log$ is involved, we will write $A B O R T(a)$ for $A B O R T(L, a)$

Definition: Let $L$ be a $\log$ in which action $a$ has not been aborted $A B O R T(a)$ is a simple abort of $a$ for $L$ if $m_{I}\left(C_{L}, A B O R T(a)\right) \neq \emptyset$ and $m_{I}\left(C_{L}, A B O R T(a)\right) \subset m_{I}\left(C_{L}-\lambda_{L}^{-1}(a)\right)$
Clearly, a simple abort of action $a$ in $\log L$ exists if and only if $m_{I}\left(C_{L}-\lambda_{L}^{-1}(a)\right)$ is a prefix of some computation of $A_{L}$ The following definitions lead to a characterisation of logs and actions for which simple aborts exist. The idea is that if we take transaction dependencies into account in aborting, then we can find a consistent set of actions to abort "via omission", and thus achieve a simple abort We first establish a notion of time relative to a given action's execution with two functions Pre and Post Then we formally define transaction dependency in terms of time ordering and conflict between actions

Notation: Given a $\log L$ and action $c \in C_{L}$, let $\operatorname{Pre}(c)$ be the partial log having concrete actions $C_{P_{\text {re }(c)}}=\left\{b \mid b \in C_{L} \wedge b<_{L} c\right\}$, abstract actions $A_{L}$, and mapping $\lambda_{P r e(c)}$ which is the restriction of $\lambda_{L}$ to the set $C_{\left.P_{\text {relc }}\right)}$ Let $C_{\text {Post(c) }}=\{b \mid b \in$ $\left.C_{L} \wedge c<_{L} b\right\}$ (Note that in general we cannot define a log Post $(c)$, because logs are defined in terms of prefixes )

The following definition says that an abstract action $b$ depends on a (non-aborted) abstract action $a$ if it has a concrete subaction which follows and conflicts with a concrete subaction of $a$ If $b$ depends on $a$, and if we restrict ourselves to simple aborts, then it may be necessary to abort $b$ when $a$ is aborted For example, suppose $b$ reads a record inserted by $a$ Merely not adding the record when redoing does not suffice to reproduce the effects of $b$ alone, since $b$ saw and could act upon the record originally provided

Definition: An action $b$ depends on an action $a$ in a $\log L$ if there is some $d \in \lambda_{L}^{-1}(b)$ and some $c \in \lambda_{L}^{-1}(a)$ such that $d$ follows $c$ in the order of $C_{L}, a$ is not aborted in the $\log \operatorname{Pre}(d)$, and $d$ and $c$ conflict

Now that we have a handle on dependencies, we introduce removabulity, a property of actions, and restorabality, a property of logs

Definition: An action $a$ of a $\log L 18$ removable If no action depends on it $A \log L$ is restorable if every aborted action is removable

Removability and restorability are intended to be descriptive (a removable action can be removed by a simple abort, re-running a restorable log without the aborted actions restores the effect of the non-aborted actions) Therr suggestiveness will be justıfied below

Restorability may be viewed as a dual condition to recoverability, which requires that no action be committed before any action whith it depends on Restorability says that no action is aborted before any action which depends on it If we do not insist on restorability, aborts may be impossible On the other, restorability implies that simple aborts always work, which we will 
show shortly.

The idea behind restorability is that we abort only actions that are at the "end" of the log (in terms of the dependency ordering, which is a partial order) at the time of the abort This notion is made more precise with the introduction of finality, which is related to removability Then we show that removability of an action implies that a simple abort of the action exists. Finally, we show the important result . if every aborted action is removable ( $1 \mathrm{e}$, the log is restorable) and the aborts are simple (accomplished by omission during redo), then the log is concretely atomic (i e , the execution is correct)

Definition: Let $C$ be a sequence of actions with order < and let $F \subset C \quad F$ is final in $C$ if for every $f \in F$ and $c \in C-F, c<f$ or $c$ and $f$ commute

Note that the set $\lambda_{L}^{-1}(a)$ is final in $C_{L}$ for any removable action $a$ It follows from this that it is the termmal subsequence of some sequence $D \approx \approx^{*} C_{L}$

Lemma 3: If action $a$ of $\log L$ is removable, then $C_{L}-\lambda_{L}^{-1}(a)$ is a prefix of a computation of $A_{L}$

Proof: We will show by induction on the number of actions in any final set $F$ of operations of $C_{L}$, that $C_{L}-F$ is a prefix of a computation The lemma then follows from the fact that $\lambda_{L}^{-1}(a)$ is final in $C_{L}$ since $a$ is removable

Induction Base ( $F$ contains only 1 action) Let $F=\{c\}$ Then $C_{L}=\gamma ; c, \delta$ for some sequences $\gamma$ and $\delta$, such that for every $d \in \delta, m(c, d)=m(d ; c)$ Hence $C_{L} \approx \gamma, \delta, c$ and therefore $C_{L}-\{c\}=\gamma, \delta$ is a prefix of a computation

Induction Hypotheszs For every final set $F$ in $C_{L}$, if $|F|<n$, then $C_{L}-F$ is a prefix of a computation of $A_{L}$

Induction Step Suppose $|F|=n$ Let $F^{\prime}=F-\{c\}$, where $c$ is the first (1 e, minimal) element of $F$ with respect to $<_{L}$ Then $F^{\prime}$ is final in $C_{L}$, so, by the induction hypothesis, $C_{L}-F^{\prime}$ is a prefix of a computation Since $c$ commutes with all later actions in $C_{L}-F^{\prime}$, we can use reasoning sumilar to the case $n=1$ to show that $C_{L}-F^{\prime} \approx^{*} C_{L}-F, c$ and therefore $C_{L}-F$ is a prefix of a computation

Since $C_{L}-\lambda_{L}^{-1}(a)$ is a prefix of a computation of $A_{L}-\{a\}$, we can restore checkpoint $I$ and rerun all actions in $C_{L}-\lambda_{L}^{-1}(a)$ in the order given by $<_{L}$ In fact, the checkpoint can be taken at any point before the initialization of $a$ Let $c$ be the first action of $a$ Let $d \in\{c\} \cup C_{P r e(c)}$ Then $C_{L}-\lambda_{L}^{-1}(a)$ is the concatenation of $C_{P r e(d)}$ and $C_{\text {Post (d) }}-\lambda_{L}^{-1}(a)$ Hence there is a state $t$ such that $(I, t) \in m_{I}\left(C_{P r e(d)}\right)$ and $m_{t}\left(C_{P \text { ost }(d)}-\lambda_{L}^{-1}(a)\right) \neq \emptyset$ Any such state $t$ can be used as a checkpoint state from which to roll forward

We now apply Lemma 3 inductively to show that if no dependencies were formed on abstract actions before they were aborted by a simple abort, then atomicity is guaranteed

Theorem 4: If $L$ is restorable and if every abort in $L$ is simple, then $L$ is atomic

Proof: Let $\left\{a_{1}, \quad, a_{n}\right\}$ be the set of aborted actions Construct the $\log M$ such that $A_{M}=A_{L}-$ $\left\{a_{1}, \quad, a_{n}\right\}, C_{M}=C_{L}-\lambda_{L}^{-1}\left(\left\{a_{1}, \quad, a_{n}\right\}\right)$, and $\lambda_{M}=\lambda_{L}$ restricted to $C_{M}$ Since $L$ is restorable, every aborted action in $L$ is removable Using Lemma 3 inductively, we see that $C_{L}-\lambda_{L}^{-1}\left(\left\{a_{1}, \ldots, a_{n}\right\}\right)$ is a prefix of a computation of $A_{M}$ This verifies that $M 18$ a $\log$

Now we must verify that $m_{I}\left(C_{L}\right)=m_{I}\left(C_{M}\right)$ To do this, we observe that there exist $\gamma_{1}, \quad, \gamma_{n+1}$ such that

$$
\begin{aligned}
C_{L}= & \gamma_{1}, \operatorname{ABORT}\left(a_{1}\right) ; \gamma_{2} ; A B O R T\left(a_{2}\right) ;, \\
& \gamma_{n}, \operatorname{ABORT}\left(a_{n}\right) ; \gamma_{n+1}
\end{aligned}
$$

The meaning of $C_{L}$ is given by

$$
\begin{aligned}
m_{I}\left(C_{L}\right)=\{\langle I, t\rangle \mid \exists u \cdot\langle(I, u\rangle & \in m_{I}\left(\beta_{1}\right) \wedge \\
\langle u, t\rangle & \left.\in m_{I}\left(\beta_{2}\right)\right\}
\end{aligned}
$$

where

$$
\begin{aligned}
& \beta_{1}=\gamma_{1}, \operatorname{ABORT}\left(a_{1}\right) \\
& \beta_{2, n}=\gamma_{2}, \operatorname{ABORT}\left(a_{2}\right), \quad, \operatorname{ABORT}\left(a_{n}\right) ; \gamma_{n+1}
\end{aligned}
$$

But by the hypothess of the theorem, every abort 18 simple, so that

$$
m_{I}\left(\gamma_{1}, A B O R T\left(a_{1}, L\right)\right) \subset m_{I}\left(\gamma_{1}-\lambda_{L}^{-1}\left(a_{1}\right)\right)
$$

and therefore

$$
m_{I}\left(C_{L}\right) \subset m_{I}\left(C_{L}-\lambda_{L}^{-1}\left(a_{1}\right)\right)
$$

Proceeding inductively, we see that

$$
\begin{aligned}
m_{I}\left(C_{L}\right) & \subset m_{I}\left(C_{L}-\lambda_{L}^{-1}\left(\left\{a_{1}, \quad, a_{n}\right\}\right)\right. \\
& =m_{I}\left(C_{M}\right) .
\end{aligned}
$$

Theorem 4 suggests a general procedure for aborting actions When an action $a$ is to be aborted, abort it and its dependent actions, namely the set of actions

$$
\operatorname{Dep}(a)=\{b \mid b \text { depends on } a\} \cup\{a\}
$$

The abort 18 done by restoring any concrete state which existed prior to the first concrete action in $\lambda_{L}^{-1}(D e p(a))$ and then re-running the actions in $C_{L}-\lambda_{L}^{-1}(D \operatorname{ep}(a))$ from that point on

\subsection{Rolling Back Actions}

A potentially much faster implementation than checkpoint/restore would simply roll back the concrete actions in the computation of an aborted action a. For this purpose, we define an $U N D O$ operator on concrete actions which chooses an inverse concrete action to perform the roll back The plan is to implement the $A B O R T$ operator on abstract actions as a sequence of UNDO actions, 
one for each concrete action called by the abstract action, applied in reverse order of execution of the concrete actions

$$
\text { UNDO } C \times S_{0} \rightarrow\left(S_{0} \rightarrow S_{0}\right)
$$

This UNDO operator chooses a state dependent inverse action which will transform the current state to the state in which the forward action was initiated Thus we must define the UNDO so that $m(c, U N D O(c, t))=$ $\{\langle t, t\rangle\}$ It follows from this definition that if $c$ is the last concrete action in $C_{L}$ and $\langle I, t\rangle \in m\left(C_{L}-\{c\}\right)$ then $m\left(C_{L}, U N D O(c, t)\right)=\{(I, t)\}$ Furthermore, if $\langle I, t\rangle \notin m\left(C_{L}-\{c\}\right)$ then $m\left(C_{L} ; U N D O(c, t)\right)=\emptyset$ In other words, if the final action $c$ was initiated in state $t$, then $U N D O(c, t)$ restores the state to $t$ and to nothing else

Actually, to undo an action $c$, it is not necessary that $c$ be the last action of $C_{L}$, only that $c$ is not followed by any action which conflicts with $U N D O(c, t)$ for the state $t$ in which $c$ was initiated This is stated in the following lemma

Lemma 4: If the following conditions all hold

$$
\begin{array}{ll}
1 & c \in C_{L} \\
2 & \langle I, t\rangle \in m\left(C_{P r e(c)}\right) \\
3 & \text { no action of } C_{P \text { ost }(c)} \text { conflicts with } \\
4 & U N D O(c, t) \notin C_{P o s t(c)} \\
\text { then } & \\
m_{I}\left(C_{L}, U N D O(c, t)\right)= \\
\quad\left\{(I, u\rangle \mid\langle t, u) \in m\left(C_{P_{\text {ost }}(c)}\right)\right\}
\end{array}
$$$$
3 \text { no action of } C_{P_{\text {ost }}(c)} \text { conflicts with } U N D O(c, t)
$$

Proof: By the definitions of $C_{P \text { re(c) }}$ and $C_{P \text { ost }(C)}$, $C_{L}=C_{P r(c)}, c, C_{P o s t(c)}$ By the hypothesis of the lemma, for every $d \in C_{P o s t(c)}$,

$$
m(d, U N D O(c, t))=m(U N D O(c, t), d)
$$

and so

$$
C_{L}, U N D O(c, t) \approx *
$$

$$
C_{\text {Pre(c) }} ; \text {; UNDO }(c, t) ; C_{\text {Post(c) }}
$$

It follows that

$$
\begin{aligned}
& m\left(C_{L}, U N D O(c, t)\right) \\
& =m\left(C_{\left.P_{\text {re }(r)}\right)} ; c, U N D O(c, t), C_{P_{\text {ont }}(c)}\right) \\
& =\{\langle s, w\rangle \mid \exists u, v \\
& \langle s, u\rangle \in m\left(C_{\text {Pre(c) }}\right) \quad \wedge \\
& \langle u, v\rangle \in m(c ; U N D O(c, t)) \wedge \\
& \langle v, w\rangle \in m\left(C_{\text {Post }(c)}\right\} \\
& =\left\{\langle s, w\rangle \mid\langle s, t\rangle \in m\left(C_{\left.P_{r e(c)}\right)}\right) \wedge\right. \\
& \left.\langle t, w\rangle \in m\left(C_{\text {Post (c) }}\right)\right\}
\end{aligned}
$$

Therefore,

$$
\begin{aligned}
& m_{I}\left(C_{L}, U N D O(c, t)\right) \\
& \quad=\left\{\langle I, u\rangle \mid\langle t, u\rangle \in m\left(C_{P_{\text {ost }}(c)}\right)\right\}
\end{aligned}
$$

The sequence of concrete actions called by an aborted abstract action $a$ in a complete $\log L$ should be a prefix $c_{1}, \quad, c_{k}$ of a computation $c_{1}, \quad, c_{n}$ of $a$, followed by $U N D O\left(c_{k}, t_{k}\right)$, , UNDO $\left(c_{1}, t_{1}\right)$ We extend the definition of concurrent computations to allow such sequences

Definition: A rolled back computation of an abstract action $a$ is a sequence

$$
c_{1}, \quad, c_{\jmath}, U N D O\left(c_{\jmath}, t_{\jmath}\right), \quad, U N D O\left(c_{1}, t_{1}\right)
$$

such that $c_{1}, \quad, c_{n}$ is a computation of $a$ and $0 \leq$ $\jmath \leq n$

Definition: A concurrent computation of a set $A=\left\{a_{1}, \quad, a_{n}\right\}$ of abstract actions is an interleaving of a set $C$ of sequences $C_{1}, \quad, C_{n}$ such that

$1 C_{1}$ is a computation or a rolled back computation of $a_{\mathbf{i}}$,

$2 m_{I}(C) \neq \emptyset$,

3 If there is an action $U N D O(c, t)$ for $c \in C$ then $\langle I, t\rangle \in m_{I}\left(C_{\text {Prelc })}\right)$

Deflnition: If an action $a$ has called an UNDO then we say that $a$ is aborted and is rolling back If it has called an UNDO for every forward action it called, then we say that $a$ is rolled back

The definition of a $\log$ is unchanged except for the expanded set of computations

Deflnition: The rollback of action a depends on action $b$ in a $\log L$ if there is a child $c$ of $a$ and a child $d$ of $b$ such that $c<_{L} d, U N D O(c, t) \notin C_{P_{\text {reld }}}$ and $U N D O(d, w) \notin C_{P r e(U N D O(c, t))}$; and $d$ conflicts with $U N D O(c, t)$

That is, $b$ interferes with the rollback of $a$ if $b$ has a nonundone child action $d$ that comes between and conflicts with a child $c$ of $a$ and the undo of $c$ We now characterize logs in which such interference does not occur

Definition: $A \log L$ is revokable of for each action $a \in A_{L}$, the rollback of $a$ does not depend on any $b \in A_{L}$

Theorem 5: If a complete $\log L$ is revokable then it is atomic

Proof: What we will show is that if $L$ is revokable then $m_{I}\left(C_{L}\right) \subset m_{I}\left(C_{M}\right)$ for the $\log M$ with

$$
\begin{aligned}
A_{M}=A_{L} & -\{a \mid a \text { is rolled back in } L\} \text { and } \\
C_{M}=C_{L} & -\left\{c \mid U N D O(c, t) \in C_{L}\right\} \\
& -\left\{U N D O(c, t) \mid t \in S_{0}\right\}
\end{aligned}
$$

( $C_{M}$ is $C_{L}$ with the undone actions and all the undos themselves deleted ) Since for a complete $\log L$ the rolled back actions are exactly the aborted ones,

$$
A_{M}=A_{L}-\{a \mid a \text { is aborted in } L\},
$$

and it follows that $L$ is atomic

The proof is by induction on the number $k$ of $U N$. $D O s$ in $C_{L}$ 
Induction Base $(k=1)$ Let $c$ be the action with $U N D O(c, t) \in C_{L}$ and let $\lambda_{L}(c)=a$ Because $L$ is revokable, there 18 no action $b$ such that the rollback of $a$ depends on $b$. In other words, for every concrete action $d$ in $C_{L}$, if $c<_{L} d<_{L} U N D O(c, t)$ then $d$ commutes with $U N D O(c, t)$ This imphes that

$$
C_{L} \approx^{*} C_{P r e(c)}, c, U N D O(c, t), C_{P o s t(c)}
$$

and therefore

$$
\begin{aligned}
& m_{I}\left(C_{L}\right) \\
& \quad=m_{I}\left(C_{P r e(c)}, c, U N D O(c, t), C_{P \text { ost }(c)}\right) \\
& \quad \subset m_{I}\left(C_{P r e(c)}, C_{P \text { ost }(c)}\right)=m_{r}\left(C_{M}\right)
\end{aligned}
$$

Induction Hypothesss If there are fewer than $k U N$ $D O_{s}$ in $C_{L}$, then $m_{I}\left(C_{L}\right) \subset m_{I}\left(C_{M}\right)$ for some log $M$ with

$$
\begin{aligned}
A_{M}=A_{L} & -\{a \mid a \text { is aborted in } L\} \\
C_{M}=C_{L} & -\left\{c \mid U N D O(c, t) \in C_{L}\right\} \\
& -\left\{U N D O(c, t) \mid t \in S_{0}\right\}
\end{aligned}
$$

Induction Step Suppose there are $k$ UNDOs in $C_{L}$ Consider the first undo, $U N D O(c, t)$, in the order $<_{L}$ Since it is the first, there is no $U N D O(d, w)$ such that $c<_{L} U N D O(d, w)<_{L} U N D O(c, t)$ Since $L$ is revokable, $U N D O(c, t)$ commutes with every action $d$ such that $c<_{L} d<_{L}$ UNDO $(c, t)$ Therefore, using the same reasoning as for the induction base, and applying the induction hypothesis,

$$
m_{I}\left(C_{L}\right) \subset m_{I}\left(C_{\left.P_{\text {relc }}\right)}, C_{P_{\text {ost }}(c)}\right) \subset m_{I}\left(C_{M}\right)
$$

If the $\log L$ is partial, we can extend $L$ to a complete log by adding $U N D O$ s for every incomplete action to the end of the log The order of the UNDOs should be the reverse of the order of the forward actions The new log 18 complete and revokable, therefore by Theorem 5 it is atomic

Theorem 5 suggests the following algorithm for aborting actions If the rollback of an action will not depend on any action in $A_{L}$, then execute a sequence of UNDOs in reverse order of the forward actions If the rollback will depend on some action, recursively abort the action on which the rollback will depend Of course, the cascaded aborts can be avorded To avold them, it is necessary to block an abstract action if a rollback dependency would develop

\subsection{Layered Atomicity}

In this section, we describe the correct aborting of actions in a multilevel system As in section 3.2, suppose that we have a system $\log \mathbf{L}=\left\{L_{1}, \quad, L_{n}\right\}$ To guarantee that the sequence of concrete actions at level $\imath+1$ 18 implemented by the abstract actions at level $\imath$, we must be able to say that there is an ordering on the nonaborted abstract actions in $A_{L_{q}}$ which is the same as the ordering on these actions when they are viewed as con- crete actions at level $\imath+1$ But this requires that each level be both serializable and atomic

Definition: Let $L$ be a complete log contanning aborted actions Let $A_{L}-\{a \mid a$ is aborted in $L\}=$ $\left\{a_{1}, \quad, a_{n}\right\} \quad L$ is abstractly serzalizable and atomic If there is a permutation $\pi$ of $\{1, \quad, n\}$ such that

$$
\rho\left(m_{I}\left(C_{L}\right)\right) \subset m_{\rho(I)}\left(a_{\pi(1)}, \quad, a_{\pi(n)}\right)
$$

$L$ is concretely serralizable and atomic if there is a permutation $\pi$ of $\{1, \quad n\}$ such that

$$
m_{I}\left(C_{L}\right) \subset m_{I}\left(\alpha_{\pi(1)}, \quad, \alpha_{\pi(n)}\right)
$$

This is simular, in combining the aspects of computational atomicity with failure atomicity, to Weihl's defin1tion of atomicity [Weihl 84] As usual, concrete serialızability and atomicity implies abstract serializability and atomicity

Definition: A system $\log L$ is abstractly sernalzzable and atomic by layers of each $\log L_{2}$ is abstractly serializable and atomic; $C_{L_{i+1}}=A_{L_{i}}-$ $\left\{a \mid a\right.$ s aborted in $\left.L_{8}\right\}=\left\{a_{2,1}, \quad ; a_{2, k_{1}}\right\} ;$ and there is a serialization order $\pi_{2}$ on level $L_{i}$ such that $C_{L_{\imath+1}}=a_{i, \pi_{2}(1) ;}, a_{i, \pi_{i}\left(k_{i}\right)}$

We now arrive at the interesting result for layered atomicity Recall that a top level log is a log relating the top level actions (in $A_{n}$ ) to the lowest level actions (in $C_{1}$ )

Theorem 6: If a system $\log \mathrm{L}$ is abstractly serializable and atomic by layers then its top level log is abstractly serializable and atomic

Proof: The proof is by induction on the number $n$ of levels

Induction Base If there is only one level, then the top level log is identical to the log for that level and is therefore abstractly serializable and atomic by the definition of layered serializability and atomicity Induction Hypothesis The top level log is abstractly serializable and atomic if the system log is abstractly serializable and atomic by layers and there are fewer than $n$ levels

Induction Step Suppose that the system log has $n$ levels By the definition of layered serializability and atomicity the level 1 log 18 abstractly serializable and atomic Therefore there 18 a $\log M$ such that $A_{M}=A_{L_{1}}-\left\{a \mid a\right.$ is aborted in $\left.L_{1}\right\}$ and

$$
\begin{aligned}
\rho_{1}\left(m_{I}\left(C_{L_{1}}\right)\right) & \subset \rho_{1}\left(m_{I}\left(C_{M}\right)\right) \\
& =m_{\rho_{1}(I)}\left(a_{1 \pi_{1}(1),}, a_{1 \pi_{1}\left(k_{1}\right)}\right)
\end{aligned}
$$

By the definition of layered serializability and atom1city $C_{L_{2}}=a_{1 \pi_{1}(1),}, a_{1 \pi_{1}\left(k_{1}\right)}$ Therefore

$$
m_{\rho_{1}(I)}\left(a_{1 \pi_{1}(1)}, \quad, a_{1 \pi_{1}\left(k_{1}\right)}\right)=m_{\rho_{1}(I)}\left(C_{L_{2}}\right)
$$

Applying the induction hypothesis to the system log M consisting of the logs $L_{2}, \quad, L_{n}$, the top level log for $M$ is abstractly serializable and atomic, that is, 
$\rho_{2}$

$$
\begin{aligned}
\circ \rho_{n} & \left(m_{\rho_{1}(I)}\left(C_{L_{2}}\right)\right) \subset \\
& m_{\rho_{1} \circ \rho_{2} \circ} \circ \rho_{n}(I) \\
& \left(C_{N}\right)
\end{aligned}
$$

for some $\log N$ with

$$
A_{N}=A_{L_{n}}-\left\{a \mid a \text { is aborted in } L_{n}\right\}
$$

It follows that

$$
\begin{gathered}
\rho_{2} \circ \quad \circ \rho_{n}\left(m_{\rho_{1}(I)}\left(C_{L_{1}}\right)\right) \subset \\
m_{\rho_{1} \circ \rho_{2} \circ \circ \rho_{n}(I)}\left(C_{N}\right)
\end{gathered}
$$

for this same $\log N$

Since revokability and restorability each imply atomicity, we immediately derive these additional results

Corollary 1 to Theorem 6: If each level of a system $\log \mathbf{L}$ is serializable and restorable, then its top level log 18 abstractly atomic

Corollary 2 to Theorem 6: If each level of a system $\log \mathbf{L}$ is serializable and revokable, then its top level $\log$ is abstractly atomic

\section{Conclusions and Further Work}

In summary, we have shown that, with respect to both serializability and fallure atomicity, the correctness of atomic actions can be assured by guaranteeing their correctness at each level of abstraction The result for serialzability alone follows from the results presented in [Beerı et al 83], but the relative simplicity of the proofs presented here is impressive

Additionally, the inclusion of decision making in the model reveals the importance of conflict based approaches to correctness of atomic actions As a consequence of Lemma 2, conflict based approaches are not only efficient, they also prevent accepting as correct certain computations which could never have occurred in a serial execution of the actions The importance of conflict in the correctness of $A B O R T$ actions and UNDO actions also seems significant

It should prove interestung to address the possibility of using different protocols for serialızability and different techniques for enforcing fallure atomicity at different levels of abstraction The implementation of such techniques for abstract actions presents a variety of problems Among the problems to be addressed is the extension of the model so that actions operate on objects rather than on the global state Also to be considered is implementation of serialization primitives such as locks and timestamps for abstract objects and implementation of recovery objects such as log entries, shadows, and intention lists at higher levels of abstraction

The relationship between forward conflict (between two actions) and backward conflict (between an action and an UNDO of another action) should also be addressed Can we implement $U N D O s$ in such a way that backward conflict occurs if and only if there is forward conflict? Also, to what extend can UNDOs be treated like ordinary actions? Can an $A B O R T$ or an UNDO be aborted or undone? What additional problems would this present?

\section{References}

[Beerı et al 83] C Beerı, P A Bernstem, N Goodman, M Y Lal, and D E Shasha, "A Concurrency Control Theory for Nested Transactions", Proc End Symp on Prin of Distributed Computing, Aug 1983, pp 45-62

[Eswaren et al 76] K P Eswaren, J N Gray, R A Lorie, I L Traiger, "The Notion of Consistency and Predicate Locks in a Database System", Comm of the ACM, Vol 19, No 11, Nov 1976, pp 624-633

[Garcia and Wiederhold 82] Hector Garcia-Molina and Gio Wiederhold, "Read-Only Transactions in a Distributed Database", Trans on Database Systems, Vol 7, No 2, June 1982, pp 209-234

[Hadzllacos 83] Vassos Hadzilacos, "An Operational Model for Database System Reliability", Proc end Symp on Prin of Database Systems, Mar 1983, Pp 244-257

[Haerder and Reuter 83] Theo Haerder and Andreas Reuter, "Principles of Transaction-Oriented Database Recovery", Computing Surveys, Vol 15, No 4, Dec 1983, pp 287-318

[Lynch 83] Nancy A Lynch, "Multilevel Atomicity - A New Correctness Criterion for Database Concurrency Control", Trans on Database Systems, Vol 8, No 4, Dec 1983, pp 484-502

[Moss et al 85] J Elıot B Moss, Nancy D Griffeth, Marc H Graham, "Abstraction in Concurrency Control and Recovery Management", Dept of Comp and Info Sc1, Univ of Mass (Amherst), Tech Report 85-51, Dec 1985

[Papadımitrıou 79] C H Papadımitrıou, "Serializability of Concurrent Updates", Journal of the $A C M$, Vol 26, No 4, Oct 1979, pp 631-653

[Schwarz and Spector 84] Peter M Schwarz and Alfred Z Spector, "Synchronizing Shared Abstract Types", Trans on Computer Systems, Vol 2, No 3 , Aug 1984, pp 223-250

[Weihl 84] William E Weihl, Specrfication and Implementation of Atomic Data Types, PhD thesis, Mass Inst of Tech, Lab for Comp Sc1 Tech Report 314, Mar 1984 